\title{
Dynamic Variation and Prognostic Value of Weight Loss During Radiotherapy in Nasopharyngeal Carcinoma: A Large-Scale Cohort Study
}

\author{
Ya-Nan Jin \\ Sun Yat-sen University Cancer Center \\ Tian-Liang Xia \\ Sun Yat-sen University Cancer Center \\ Dong-Mei Mai
}

Sun Yat-sen University Cancer Center

Ji-Jin Yao

Sun Yat-sen University Cancer Center

Chang Jiang

Sun Yat-sen University Cancer Center

Wen-Zhuo He

Sun Yat-sen University Cancer Center

Liangping Xia ( $\nabla$ xialp@sysucc.org.cn )

Sun Yat-sen University Cancer Center https://orcid.org/0000-0003-0104-7700

\section{Research article}

Keywords: Nasopharyngeal carcinoma, radiotherapy, weight loss, dynamic variation, prognostic value.

Posted Date: February 12th, 2021

DOl: https://doi.org/10.21203/rs.3.rs-198420/v1

License: (9) This work is licensed under a Creative Commons Attribution 4.0 International License. Read Full License 


\section{Abstract}

Background: We aim to investigate the dynamic variation and prognostic value of weight loss among patients with nasopharyngeal carcinoma (NPC).

Methods: A total of 1149 newly diagnosed NPC patients who received radical radiotherapy (RT) were retrospectively analyzed. Patients' weights were measured at initiation of RT and every week during RT. Recursive partitioning analyses (RPAs) were utilized to determine cut-off value for rate of weight loss (RWL). Disease-free survival (DFS) was our primary endpoint. Secondary endpoints included locoregional relapse-free survival (LRRFS), distant metastasis-free survival (DMFS), and overall survival (OS).

Results: RWLs were $0 \%, 0 \%,-1.54 \%,-2.86 \%,-4.11 \%,-5.98 \%,-6.56 \%$ at $1^{\text {st }}, 2^{\text {nd }}, 3^{\text {rd }}, 4^{\text {th }}, 5^{\text {th }}, 6^{\text {th }}$, and $7^{\text {th }}$ week of RT, respectively. RWL optimal threshold with respect to DFS was $-5.3 \%$ based on RPAs. Thus, a consistent threshold of $-5 \%$ (> $-5 \%$ versus $\leq-5 \%$ ) was selected to classify NPC patients into low RWL and high RWL groups for survival analysis. Compared to high RWL ( $\leq-5 \%)$, patients with low RWL $(>-5 \%)$ had significantly better ten-year DFS (78.6\% versus $61.2 \%$; $P<0.001)$, OS ( $86.6 \%$ versus $70.1 \% ; P<0.001)$, and DMFS (88.5\% versus $80.2 \% ; P=0.007)$. However, no difference was observed between LRRFS groups (91.7\% versus $94.3 \% ; P=0.173$ ). In multivariate analysis, high $R W L$ was an independent risk factor for DFS (HR, 1.56; 95\% Cl, 1.19-2.03; $\mathrm{P}=0.001)$, OS (HR, 1.54; 95\% Cl, 1.11-2.15; $\mathrm{P}=0.011)$ and DMFS (HR, $1.47 ; 95 \% \mathrm{Cl}, 1.03-2.10 ; \mathrm{P}=0.033)$ in patients with NPC. Additionally, chemotherapy and old age were attributed to the development of high RWL.

Conclusions: Weight loss during RT was significantly associated with decreased survival among NPC patients. Clinicians should continuously inform patients on the health impact of minimizing weight loss under $5 \%$.

\section{Background}

Nasopharyngeal carcinoma (NPC), an epithelial malignancy that is distinguished from other head and neck cancers, is highly prevalent in southern China [1]. The main treatment for NPC is radiotherapy (RT) due to anatomical restrictions and radio-sensitivity. Over the past decade, advances in imagining techniques, chemotherapy, and radiation technology contributed to the improved NPC survival. However, $20-30 \%$ of patients still die as a result of NPC recurrence [2-3]. Therefore, efforts to identify modifiable risk factors can potentially provide new insights on developing clinical intervention for increasing longterm survival.

Patients diagnosed with head and neck cancers often experience weight loss during treatment due to acute toxicity, such as mucositis, dysgeusia, xerostomia, and nausea [4-7]. Previous studies [8,9] have estimated weight loss to range from 40-90\%, especially among NPC patients where rates are high. Substantial weight loss during treatment was associated with poor survival in NPC [10-13]. Monitoring the decreasing weight during RT will allow for clinicians to evaluate the current treatment plan effectiveness for NPC [14]. Moreover, knowing the dynamic variation of patients' weight loss during 
treatment is helpful in selecting the optimal time for nutritional intervention and altering RT treatment. However, bodyweight was only obtained at baseline visit and again at the end of treatment in previous studies [10-12], without considering the dynamic weight loss during treatment.

To fill the current gaps in knowledge and limitations of previous studies, we conducted a large-scale retrospective study of NPC patients treated with radical RT. The present study sought to (1) draw a dynamic map of weight variation during RT; (2) identify the weight loss prognostic value on survival outcomes; (3) demonstrate risk factors for weight loss; and (4) provide insight on individualized nutritional intervention and the timing of RT replanning for NPC patients.

\section{Methods}

\section{Patient characteristics}

The present study was a retrospective cohort study utilizing in-patient medical records from Sun Yat-Sen University Cancer Center consisting of first diagnosis of histologically confirmed, non-disseminated NPC from January 2006 to October 2014. We included patients if they met the following criteria: (1) newly diagnosed non-disseminated NPC; (2) Karnosfky performance score $\geq 70$; (3) no indication of distant metastases; (4) absent of secondary malignancy; (5) treated with radical intensity-modulated radiation therapy (IMRT); and (6) weekly assessment of weight. This study was conducted in compliance with institutional policy to protect patients' private information, and was approved by the Institutional Review Board of our center.

We included 1149 patients, and the baseline assessment included full physical examination, fiberoptic nasopharyngoscopy, neck and nasopharyngeal magnetic resonance imaging (MRI), computed tomography (CT), abdominal ultrasonography, biochemistry profiling and hematology, whole body bone scan (ECT) or ${ }^{18}$ F-fluorodeoxyglucose positron emission tomography and CT (PET-CT). Real-time quantitative polymerase chain reaction was used to measure Epstein-Barr virus (EBV) DNA concentrations as previously described in detail [15]. Patients were staged based on the 7th edition of the American Joint Commission on Cancer staging system [16].

\section{Radiotherapy and chemotherapy}

All patients received radical IMRT in the current study. Dose prescribed to patients were (1) $66-70$ Gy at 2.12-2.27 Gy/fraction to planning target volume (PTV) of nasopharyngeal gross tumor volume (GTVnx); (2) PTV of GTV of the metastatic lymph nodes (GTVnd) received 64-70 Gy; and (3) high-risk clinical target volume (CTV1) received 60-63 Gy to PTV and (4) low-risk clinical target volume (CTV2) received 50-56 Gy to PTV. Concurrent chemotherapy comprised of cisplatin ( 80 or $100 \mathrm{mg} / \mathrm{m}^{2}$ ) given in weeks one, four, and seven of radiotherapy, or cisplatin $(40 \mathrm{mg} / \mathrm{m} 2)$ given weekly during radiotherapy. Induction chemotherapy (IC) included cisplatin $\left(60 \mathrm{mg} / \mathrm{m}^{2}\right)$, docetaxel $\left(60 \mathrm{mg} / \mathrm{m}^{2}\right)$, and 5 -fluorouracil $\left(600 \mathrm{mg} / \mathrm{m}^{2} /\right.$ day over $\left.120 \mathrm{~h}\right)$, or cisplatin $\left(80 \mathrm{mg} / \mathrm{m}^{2}\right)$ plus 5 -fluorouracil $\left(800 \mathrm{mg} / \mathrm{m}^{2} /\right.$ day over $\left.120 \mathrm{~h}\right)$ or cisplatin $\left(80 \mathrm{mg} / \mathrm{m}^{2}\right)$ plus docetaxel $\left(80 \mathrm{mg} / \mathrm{m}^{2}\right)$ every three weeks for three cycles. 


\section{Data collection}

Patients' age, height, weight, sex, pre-therapy laboratory counts of serum lactate dehydrogenase (LDH), high sensitivity C-reactive protein (hs-CRP), plasma EBV DNA, pathological types, clinical stage, and treatment type were extracted from medical records. Digital electronic scale (XiangShan, EB9871) was used to measure bodyweight to the nearest $0.1 \mathrm{~kg}$ in light garment and without shoes. We measured patients' bodyweight at initiation of RT and every week during RT. Bodyweight before RT ( $\mathrm{W}_{\text {Pre-RT }}$ ) was measured at initiation of RT, and $\mathrm{W}_{\mathrm{RT} 1,2,3,4,5,6,7}$ (body weight at 1st, 2nd, 3rd, 4th, 5th, 6th, and 7th week of RT) was measured at each week of RT. RWL $\mathrm{RW}_{1,2,3,4,5,6,7}$ was calculated using the following equation: $\left(\mathrm{W}_{\mathrm{RT} 1,2,3,4,5,6,7}-\mathrm{W}_{\text {Pre-RT }}\right) / \mathrm{W}_{\text {Pre-RT }} \times 100 \%$. At time of study, all patients were on $100 \%$ oral intake, where no type of enteral feeding tube or total parental nutrition were used.

\section{Follow-up and endpoints}

Patients were examined every three months during the first two years, and every six months for years three through five, and annually thereafter until death. Disease-free survival (DFS) was our primary endpoint, defined as time from diagnosis to documented recurrence of disease (either distant metastasis or locoregional disease recurrence) or mortality from any cause, whichever occurred first. Secondary endpoints consisted of (1) distant metastasis free survival (DMFS) (no documented distant metastasis); (2) locoregional relapse free survival (LRRFS) (no documented locoregional recurrence); and (3) overall survival (OS).

\section{Statistical methods}

In this study covariates included host factors (e.g. age, gender, tobacco smoking status, hs-CRP, LDH, and plasma EBV DNA), treatment factors (e.g. treatment modality), and tumor factors (e.g. histology, T stage, and $\mathrm{N}$ stage). We classified categorical variables based on clinical findings. Determined by routine cutoff points and findings from prior studies, continuous variables were converted to categorical variables [1719]. $\chi^{2}$ test or Fisher's Exact test were used to compare clinicopathologic characteristics groups.

Kaplan-Meier method was used to calculate actuarial rates, where we compared differences by the logrank test. Optimal RWL threshold was identified by performing recursive partitioning analyses (RPAs) for the cohort. Univariable with a $\mathrm{P}<0.05$ was used to include covariates in the multivariable model. Multivariate Cox proportional hazards model was applied to evaluate the impact of RWL on survival outcomes. Statistical tests were two-tailed and $\mathrm{P}<0.05$ was considered statistically significant. All statistical analysis and generate figures were performed by the rms package in $\mathrm{R}$ version 3.3.2 (http://www.r-project.org/).

\section{Results}

\section{Patient characteristics}


Clinicopathological characteristics of the 1149 patients are shown in Table 1. Among all patients, 892 $(77.6 \%)$ were male and majority $(1058,92 \%)$ of patients experienced weight loss during RT. The percentage of patients at stage I, II, III, and IVA-B were $2.1 \%, 9.9 \%, 53.7 \%$, and $34.3 \%$, respectively. Additionally, $140(12 \%)$ received RT alone and 1009 (88\%) received chemotherapy. Of these patients receiving chemotherapy, 549 (48\%; 549/1009) received IC plus CCRT and 460 (40\%; 460/1009) received CCRT alone. The median follow-up time was 72.6 months (54.8, 6-85.8 months). 
Table 1

Baseline patient characteristics according to rate of weight loss (RWL).

\begin{tabular}{|c|c|c|c|c|}
\hline Characteristic & Total $(\mathrm{N}=1149)^{\mathrm{a}}$ & No. (\%) of patients b & RWL & $P$ value \\
\hline & & $>-5 \%(n=402,35 \%)$ & $\leq-5 \%(n=747,65 \%)$ & \\
\hline Gender & & & & 0.826 \\
\hline Male & $892(77.6)$ & $314(78.1)$ & $578(77.4)$ & \\
\hline Female & $257(22.4)$ & $88(21.2)$ & $169(22.6)$ & \\
\hline Age, y & & & & 0.006 \\
\hline$<30$ & $68(5.9)$ & $28(7.0)$ & $40(5.4)$ & \\
\hline $30-39$ & $263(22.9)$ & $96(23.9)$ & $167(22.4)$ & \\
\hline $40-49$ & 447 (38.9) & $143(35.6)$ & 304 (40.7) & \\
\hline $50-59$ & $227(19.8)$ & $67(16.7)$ & $160(21.4)$ & \\
\hline$\geq 60$ & $144(12.5)$ & 68 (16.9) & $76(10.2)$ & \\
\hline Histology (WHO) & & & & 0.732 \\
\hline Type I-II & $35(3.1)$ & $11(2.7)$ & $24(3.2)$ & \\
\hline Type III & $1114(96.9)$ & 391 (97.3) & $723(96.8)$ & \\
\hline Smoking history & & & & 0.996 \\
\hline No & $807(70.2)$ & $282(70.2)$ & $525(70.3)$ & \\
\hline Yes & $342(29.8)$ & $120(29.9)$ & $222(29.7)$ & \\
\hline T stage (7th edition) & & & & 0.031 \\
\hline T1 & $71(6.2)$ & $32(8.0)$ & $39(5.2)$ & \\
\hline T2 & $185(16.1)$ & $69(17.2)$ & $116(15.5)$ & \\
\hline T3 & $627(54.6)$ & $226(56.2)$ & 401 (53.7) & \\
\hline T4 & $266(23.2)$ & 75 (18.7) & $191(25.6)$ & \\
\hline $\mathrm{N}$ stage (7th edition) & & & & $<0.001$ \\
\hline NO & $128(11.1)$ & $59(14.7)$ & $69(9.2)$ & \\
\hline
\end{tabular}

Abbreviations: RWL, rate of weight loss; WHO, World Health Organization; hs-CRP, high sensitivity Creactive protein; LDH, lactate dehydrogenase; EBV, Epstein-Barr virus;

a Percentages may not add up to 100 due to rounding.

${ }^{\mathrm{b}}$ All variables were measured before treatment. 


\begin{tabular}{|c|c|c|c|c|}
\hline Characteristic & Total $(N=1149)^{a}$ & No. (\%) of & RWL & $P$ value \\
\hline N1 & $448(39.0)$ & $177(44.0)$ & $271(36.3)$ & \\
\hline N2 & 399 (34.7) & $118(29.4)$ & $281(37.6)$ & \\
\hline N3 & $174(15.1)$ & 48 (11.9) & $126(16.9)$ & \\
\hline Overall stage & & & & 0.001 \\
\hline Stage I & $24(2.1)$ & $13(3.2)$ & $11(1.5)$ & \\
\hline Stage II & $114(9.9)$ & 53 (13.2) & $61(8.2)$ & \\
\hline Stage III & $617(53.7)$ & $223(55.5)$ & $394(52.7)$ & \\
\hline Stage IVA-B & $394(34.3)$ & $113(28.1)$ & $281(37.6)$ & \\
\hline hs-CRP, g/mL ${ }^{b}$ & & & & 0.536 \\
\hline$<1.0$ & $363(31.6)$ & $133(33.1)$ & $230(30.8)$ & \\
\hline $1.0-3.0$ & $370(32.2)$ & $132(32.8)$ & $238(31.9)$ & \\
\hline$\geq 3.0$ & $416(36.2)$ & $137(34.1)$ & $279(37.4)$ & \\
\hline $\mathrm{LDH}, \mathrm{U} / \mathrm{L}^{\mathrm{b}}$ & & & & 0.095 \\
\hline$<245$ & $1058(92.1)$ & $378(94.0)$ & $680(91.0)$ & \\
\hline$\geq 245$ & 91 (7.9) & $24(6.0)$ & $67(9.0)$ & \\
\hline EBV DNA, copy $/ \mathrm{mL}^{\mathrm{b}}$ & & & & 0.043 \\
\hline$<1,000$ & $500(43.5)$ & $193(48.0)$ & 307 (41.1) & \\
\hline $1,000-9,999$ & $266(23.2)$ & $96(23.9)$ & $170(22.8)$ & \\
\hline $10,000-99,999$ & $268(23.3)$ & $82(20.4)$ & $186(24.9)$ & \\
\hline$\geq 100,000$ & $115(10.0)$ & $31(7.7)$ & $84(11.2)$ & \\
\hline Treatment & & & & $<0.001$ \\
\hline RT alone & $140(12.2)$ & $81(20.2)$ & $59(7.9)$ & \\
\hline CCRT alone & $460(40.0)$ & $126(31.3)$ & 334 (44.7) & \\
\hline
\end{tabular}

Abbreviations: RWL, rate of weight loss; WHO, World Health Organization; hs-CRP, high sensitivity Creactive protein; LDH, lactate dehydrogenase; EBV, Epstein-Barr virus;

a Percentages may not add up to 100 due to rounding.

${ }^{\mathrm{b}}$ All variables were measured before treatment. 


\begin{tabular}{|c|c|c|c|c|}
\hline \multirow{2}{*}{$\begin{array}{l}\text { Characteristic } \\
\text { IC + CCRT }\end{array}$} & \multirow{2}{*}{$\begin{array}{l}\text { Total }(\mathrm{N}=1149)^{\mathrm{a}} \\
549(47.8)\end{array}$} & \multicolumn{2}{|c|}{ No. (\%) of patients by RWL } & \multirow[t]{2}{*}{$P$ value } \\
\hline & & $195(48.5)$ & $354(47.4)$ & \\
\hline \multicolumn{5}{|c|}{$\begin{array}{l}\text { Abbreviations: RWL, rate of weight loss; WHO, World Health Organization; hs-CRP, high sensitivity C- } \\
\text { reactive protein; LDH, lactate dehydrogenase; EBV, Epstein-Barr virus; }\end{array}$} \\
\hline \multicolumn{5}{|c|}{ a Percentages may not add up to 100 due to rounding. } \\
\hline
\end{tabular}

\section{Identification of the optimal cut-off value for defining RWL as high or low}

Five- and ten-year DFS, OS, DMFS, and LRRFS rates were $76.3 \%$ and $66.8 \%, 86.0 \%$ and $75.3 \%, 85.9 \%$ and $83.1 \%$, and $93.2 \%$ and $92.7 \%$, respectively. For the RWL, the optimal cutoff point for DFS among the entire group was $-5.3 \%$ based on RPAs. Thus, a uniform cutoff point was selected at $-5 \%$ ( $>-5 \%$ versus $\leq-5 \%$ ) to classify patients into groups low RWL and high RWL for survival analysis. 63\% (725/1149) of patients suffered $\geq 5 \%$ weight loss. When comparing survival between groups, our findings showed patients with high RWL had poorer ten-year DFS (61.2\% versus 78.5\%; $\mathrm{P}<0.001$; Fig. $1 \mathrm{~A})$, $0 \mathrm{~S}(70.1 \%$ versus $86.6 \%$; $\mathrm{P}<$ 0.001 ; Fig. 1B), and DMFS (80.2\% versus $88.5 \%$; $P=0.007$; Fig. $1 \mathrm{C}$ ) compared to low RWL patients. However, no associated difference between groups for ten-year LRRFS (91.7\% versus $94.3 \% ; P=0.173$; Fig. 1D) was observed. Further analysis revealed that high RWL was an independent unfavorable prognostic factor for DFS (HR, 1.56; 95\% Cl, 1.19-2.03; $\mathrm{P}=0.001)$, OS $(\mathrm{HR}, 1.54 ; 95 \% \mathrm{Cl}, 1.11-2.15 ; \mathrm{P}=$ $0.011)$ and DMFS (HR, 1.47; $95 \% \mathrm{Cl}, 1.03-2.10 ; \mathrm{P}=0.033)$ in multivariate analysis (Table 2$)$. 
Table 2

Multivariate analysis of prognostic factors for patients with NPC $(n=1149)$.

\begin{tabular}{|c|c|c|c|c|}
\hline Endpoint & Variable & HR & 95\% Cl for HR & $P^{a}$ \\
\hline \multicolumn{5}{|l|}{ DFS } \\
\hline & RWL status & 1.56 & $1.19-2.03$ & 0.001 \\
\hline & Age & 1.36 & $1.08-1.71$ & 0.010 \\
\hline & Gender & 0.83 & $0.63-1.08$ & 0.157 \\
\hline & Pathology & 0.44 & $0.26-0.72$ & 0.001 \\
\hline & T stage & 1.62 & $1.16-2.27$ & 0.005 \\
\hline & $\mathrm{N}$ stage & 1.54 & $1.20-1.97$ & 0.001 \\
\hline & EBV DNA & 1.50 & $1.12-2.01$ & 0.007 \\
\hline & Treatment strategy & 0.84 & $0.54-1.32$ & 0.455 \\
\hline
\end{tabular}

OS

\begin{tabular}{llll} 
RWL status & 1.54 & $1.11-2.15$ & 0.011 \\
\hline Age & 1.57 & $1.17-2.10$ & 0.002 \\
\hline Pathology & 0.37 & $0.21-0.66$ & 0.001 \\
\hline T stage & 1.84 & $1.20-2.81$ & 0.005 \\
\hline N stage & 1.72 & $1.26-2.81$ & 0.001 \\
\hline Smoking history & 1.26 & $0.93-1.70$ & 0.131 \\
\hline LDH & 1.27 & $0.80-2.03$ & 0.308 \\
\hline EBV DNA & 1.54 & $1.08-2.21$ & 0.018
\end{tabular}

DMFS

\begin{tabular}{|llll|} 
RWL status & 1.47 & $1.03-2.10$ & 0.033 \\
\hline Age & 1.43 & $1.04-1.96$ & 0.026 \\
\hline Pathology & 0.55 & $0.27-1.13$ & 0.104 \\
\hline T stage & 1.41 & $0.92-2.18$ & 0.118 \\
\hline
\end{tabular}

Abbreviations: RWL, rate of weight loss; $\mathrm{HR}$, hazard ratio; $95 \% \mathrm{Cl}, 95 \%$ confidence interval; DFS, disease-free survival; OS, overall survival; DMFS, distant metastasis-free survival; LRRFS, locoregional relapse-free survival; LDH, lactate dehydrogenase; EBV, Epstein-Barr virus.

a $P$ values were calculated using an adjusted Cox proportional hazards model. 


\begin{tabular}{|c|c|c|c|c|}
\hline Endpoint & Variable & HR & $95 \% \mathrm{Cl}$ for $\mathrm{HR}$ & $p^{a}$ \\
\hline & N stage & 1.99 & $1.41-2.80$ & $<0.001$ \\
\hline & Smoking history & 1.37 & $0.99-1.90$ & 0.055 \\
\hline & LDH & 1.33 & $0.82-2.16$ & 0.247 \\
\hline & EBV DNA & 2.01 & $1.23-3.30$ & 0.006 \\
\hline \multicolumn{5}{|l|}{ LRRFS } \\
\hline & Pathology & 0.31 & $0.14-0.72$ & 0.006 \\
\hline & Smoking history & 0.56 & $0.31-1.01$ & 0.056 \\
\hline & Treatment strategy & 4.86 & $1.17-20.15$ & 0.029 \\
\hline \multicolumn{5}{|c|}{$\begin{array}{l}\text { Abbreviations: RWL, rate of weight loss; HR, hazard ratio; } 95 \% \mathrm{Cl}, 95 \% \text { confidence interval; DFS, } \\
\text { disease-free survival; OS, overall survival; DMFS, distant metastasis-free survival; LRRFS, locoregiona } \\
\text { relapse-free survival; LDH, lactate dehydrogenase; EBV, Epstein-Barr virus. }\end{array}$} \\
\hline \multicolumn{5}{|c|}{ a $P$ values were calculated using an adjusted Cox proportional hazards model. } \\
\hline
\end{tabular}

The dynamic variation of weight loss during RT was analyzed. We observed that RWL remained largely unchanged in the first two weeks of RT, and then began to drop at a relatively stable rate during weeks three to five of RT (from- $1.54 \%$ to $-4.11 \%$; at a rate of $-1.3 \%$ weight loss per week). At the 6 th week of RT, the RWL dropped faster than the prior week (from $-4.11 \%$ to $-5.98 \%$; nearly $2 \%$ weight loss in this week). RWL then slowed in the last week of RT (from $-5.98 \%$ to $-6.56 \%$; approximately $0.5 \%$ weight loss in this week). The median weight loss during RT was $-4.0 \mathrm{~kg}(\mathrm{IQR},-2.0$ to $-6.0 \mathrm{~kg})$ and the percentage for median weight loss was $-6.7 \%$ (IQR, -3.5 to $-9.7 \%)$.

\section{Correlation between RWL and clinicopathological characteristics}

Table 1 presents the correlations between clinicopathological characteristics and RWL. High RWL patients were more likely to have advanced TNM stage (advanced T, N, and/or overall stage) $(\mathrm{P}<0.05$ for all). With respect to treatment strategy, the proportion receiving RT alone among the low RWL patient group was associated with higher receipt compared with high RWL $(20.2 \%$ versus $7.9 \% ; P<0.001)$. Factors associated with development of high or low RWL were analyzed. After logistic regression analysis, treatment modality and age remained associated with high RWL $(\mathrm{P}<0.05$ for all; Table 3$)$. Patients treated with RT alone had the lowest risk with development of weight loss during RT. In contrast, CCRT alone had a significantly strong correlation with the development of weight loss in comparison to RT alone or IC followed by CCRT (OR, 1.71; $95 \% \mathrm{Cl}, 1.23-2.31 ; \mathrm{P}<0.001)$. Moreover, patients older than 45 were more likely to suffer high weight loss than patients age $\leq 45(\mathrm{OR}, 1.35 ; 95 \% \mathrm{Cl}, 1.12-1.67 ; \mathrm{P}=0.002)$ during RT. 
Table 3

Multivariate analysis of prognostic factors for the development of high RWL in patients with NPC.

\begin{tabular}{llll}
\hline Variable & HR & $95 \%$ Cl for HR & $P$ valu \\
\hline Age at diagnosis, y & & & \\
\hline$\leq 45$ & Reference & & 0.023 \\
\hline$>45$ & 2.03 & $1.13-3.66$ & \\
\hline T stage & & & 0.908 \\
\hline T1 & Reference & & 0.625 \\
\hline T2 & 0.96 & $0.53-1.73$ & 0.220 \\
\hline T3 & 0.87 & $0.50-1.51$ & \\
\hline T4 & 1.46 & $0.80-2.68$ & \\
\hline
\end{tabular}

N stage

NO

$\begin{array}{llll}\text { N1 } & 1.01 & 0.66-1.55 & 0.952 \\ \text { N2 } & 1.52 & 0.95-2.44 & 0.075 \\ \text { N3 } & 1.68 & 0.97-2.91 & 0.064\end{array}$

$\mathrm{LDH}, \mathrm{U} / \mathrm{L}^{\mathrm{b}}$

$<245$

Reference

$\geq 245$

1.38

$0.83-2.29$

0.232

EBV DNA, copy $/ \mathrm{mL}^{\mathrm{b}}$

$\begin{array}{llll}<1,000 & \text { Reference } & & \\ 1,000-9,999 & 0.91 & 0.65-1.28 & 0.604 \\ 10,000-99,999 & 1.11 & 0.78-1.57 & 0.586 \\ \geq 100,000 & 1.30 & 0.79-2.12 & 0.315\end{array}$

Treatment

Abbreviations: $\mathrm{HR}$, hazard ratio; $95 \% \mathrm{Cl}, 95 \%$ confidence interval; $\mathrm{LDH}$, lactate dehydrogenase; hs-CRP, high sensitivity C-reactive protein; RT, radiotherapy; CCRT, concurrent chemoradiotherapy; IC + CCRT, induction chemotherapy plus concurrent chemoradiotherapy.

a $P$ values were calculated using an adjusted Cox proportional hazards model.

${ }^{\mathrm{b}}$ All variables were measured before treatment. 


\begin{tabular}{|llll|}
\hline Variable & HR & 95\% Cl for HR & Pvalue $^{\text {a }}$ \\
\hline RT alone & Reference & & \\
\hline CCRT alone & 3.29 & $2.18-4.96$ & 0.001 \\
\hline IC + CCRT & 2.03 & $1.32-3.13$ & 0.001 \\
\hline $\begin{array}{l}\text { Abbreviations: HR, hazard ratio; } 95 \% \\
\text { high sensitivity C-reactive protein; RT, radiotherapy; CCRT, concurrent chemoradiotherapy; IC + CCRT, } \\
\text { induction chemotherapy plus concurrent chemoradiotherapy. }\end{array}$ & \\
\hline a $P$ values were calculated using an adjusted Cox proportional hazards model. & \\
\hline b All variables were measured before treatment. & \\
\hline
\end{tabular}

\section{Discussion}

To the best of our knowledge, this study is the longest follow-up analysis for dynamic variation of weight loss during RT. Results of this present study revealed body weight remained largely unchanged during RT for the first two weeks, and dropped fastest at the sixth week of RT. Further analysis revealed that weight loss $\geq 5 \%$ during RT was associated with significantly inferior ten-year DFS, OS, and DMFS for NPC. Additionally, older age and chemotherapy were predictive of greater weight loss for NPC.

Numerous studies have demonstrated that weight loss is correlated with poor prognosis among individuals diagnosed with head and neck cancer [20, 21], especially among NPC patients $[10,11]$. Results from the present study aligned closely with prior studies. There are several potential reasons for these findings. First, critical weight loss may result in loosening of posture fixation, inaccurate radiation field, and significant dosimetric change during IMRT [22]. Second, weight loss potentially causes reduction in treatment tolerance and radiotherapy breaks, thus influencing therapeutic efficacy $[23,24]$. Third, weight loss is often used as a tool for assessment of newly developed malnutrition, which contributes to weakness in immunity defense mechanism, such as phagocyte function, anatomic barriers, cellular and humoral immunity. Therefore, increasing infection susceptibility and reduced response to malignancy $[25,26]$.

Previous studies reported that weight loss $\geq 5 \%$ during RT was associated with poor survival [12, 23]. Our results showed that RWL $\leq-5 \%$ was associated with poor DFS, OS, and DMFS, aligning with prior studies. However, Du et al. [11] assessed weight loss during the entire treatment procedure and observed weight loss $\geq 10 \%$ was an indicator for likelihood of metastasis and survival. One reason underlying this inconsistency cutoff value of RWL may partly be due to potential selection bias in the study population. Collectively, the impact of weight loss on prognosis of NPC can be determined in the present study. Previous literatures $[27,28]$ reported approximately $90 \%$ local control rates for NPC in the IMRT era. Due to recent advances in RT technology, no observed associated differences in LRRFS between patients in high and low RWL groups were found in the present study. These finding are reasonable as excellent 
locoregional control is expected for IMRT, therefore actual impact of weight loss on LRRFS would be limited.

Numerous factors may influence weight loss among cancer patients. We observed that patients treated with chemotherapy suffered more weight loss, similar with findings by Qiu et al. [9] and Du et al. [11]. Although encouraging results attained by multimodal therapy for NPC, acute toxicities are more likely to occur during aggressive chemoradiotherapy [9], including severe oral mucositis, nausea, and vomiting. Additionally, we observed that patients aged $>60$ years had a greater likelihood of suffering higher weight loss. We speculate that elderly patients' reduced organ reserve, multiple comorbidities, and poor treatment tolerance explain the reason for greater weight loss. Other risk factors including radiation technique, segmentation model, and prescribed dose are partly relevant to oral mucositis and weight loss. Because this research adopts the unified radiation technique, segmentation model, and prescribed dose, we did not include the above factors for analysis at last.

Since weight loss is common among NPC patients, it is necessary to assess weight change over RT time. This is due to potentially providing a more complete understanding on the relationship between bodyweight and survival among NPC patients. However, prior researches primarily evaluated weight loss at a single time point, usually pre- or post-treatment. For this reason, limited knowledge exists about the dynamic change of weight loss during RT. Our results indicated that bodyweight remained generally unchanged in the first two weeks of RT, and then began to drop relatively stable the next three weeks, though fastest in the sixth week of RT. The following reasons may explain the observed results. First, the oral mucous membrane reaction of patients is mild and diet is less affected in the first two weeks of RT. With the increasing number of RT, weight loss is gradually accelerated due to oral mucositis, aggravated swallowing pain, and decreased treatment tolerance [4].

There are some limitations should be noted. First, we lacked detailed information on dietary habit and food intake, as well as data on nutritional status was unavailable for further analysis of weight loss. However, during the study period, no standard criteria for nutritional support in patients undergoing RT has been established. Second, the data used in this study derived from only one institution in an endemic area, where a large proportion of physicians have expertise in diagnosing and treating of NPC. Future studies that incorporate external validation is needed.

\section{Conclusions}

In conclusion, our findings revealed weight loss decreased fastest during the sixth week of RT. The optimal threshold for RWL adversely impacting NPC prognosis was $-5 \%$. More efforts are needed to be given towards limiting weight loss during RT under $5 \%$ in clinical practice as a result of the detrimental impact of RWL on survival outcomes. In addition, use of chemotherapy and old age were attributed to the development of high weight loss. These findings would be helpful in selecting optimal time for nutritional intervention and RT replanning. 


\section{Abbreviations}

NPC: Nasopharyngeal carcinoma; RT: Radiotherapy; IMRT: RWL: Rate of weight loss; Intensity-modulated radiation therapy; MRI: Magnetic resonance imaging; CT: Computed tomography; ECT: Whole body bone scan; PET-CT: 18F-fluorodeoxyglucose positron emission tomography and CT; EBV: Epstein-Barr virus; PTV: Planning target volume; GTVnx: Nasopharyngeal gross tumor volume; GTVnd: GTV of the metastatic lymph nodes; CTV1: High-risk clinical target volume; CTV2: Low-risk clinical target volume; IC: Induction chemotherapy; CCRT: Concurrent chemoradiotherapy; LDH: Lactate dehydrogenase; hs-CRP: High sensitivity C-reactive protein; WPre-RT: Bodyweight before RT; DFS: Disease-free survival; DMFS: Distant metastasis free survival; LRRFS: Locoregional relapse free survival; OS: Overall survival; RPAs: Recursive partitioning analyses;

\section{Declarations}

\section{Acknowledgements}

The authors acknowledge the contributions of all of the study participants.

\section{Funding}

This work was supported by grants from the National Natural Science Foundation of China (81901699, 81773051, and 81572409) and Guangdong Medical Science and Technology Research Fund (C2018063). The funders had no role in study design, data collection and analysis, decision to publish, or preparation of the manuscript.

\section{Availability of data and materials}

The datasets analysed during the current study are available in the Research Data Deposit (RDD) public platform www.researchdata.org.cn, with the approval RDD number of RDDA2019001296. If someone need to access the data, he/she should obtain our consent, and have to explain the source of the data in their study.

\section{Authors' contributions}

Data collection and writing original draft was performed by JYN, XTL, and MDM. Formal analysis, reviewing and editing was done by YJJ and JC. JYN and XTL participated in project administration and study design. Study design was conceived and designed by XLP and HWZ. Prior to submission of this manuscript, all authors have reviewed and approved.

\section{Ethics approval and consent to participate}

This study was conducted in compliance with institutional policy to protect patients' private information, and was approved by the Institutional Review Board of Sun Yat-sen University Cancer Center. As the 
current study was a retrospective assessment of routine data, the ethics committee of our Cancer Center waived the need for individual informed consent.

\section{Consent for publication}

Not applicable.

\section{Competing interests}

The authors declare that they have no competing interests.

\section{References}

1. Wee JT, Ha TC, Loong SL, Qian CN. Is nasopharyngeal cancer really a "Cantonese cancer"? Chin J Cancer. 2010;29(5):517-26.

2. Mao YP, Tang LL, Chen L, Sun Y, Qi ZY, Zhou GQ, et al. Prognostic factors and failure patterns in nonmetastatic nasopharyngeal carcinoma after intensity-modulated radiotherapy. Chin J Cancer. 2016;35(1):103.

3. Sun XS, Liu SL, Luo MJ, Li XY, Chen QY, Guo SS, et al. The Association Between the Development of Radiation Therapy, Image Technology, and Chemotherapy, and the Survival of Patients with Nasopharyngeal Carcinoma: A Cohort Study From 1990 to 2012. Int J Radiat Oncol Biol Phys. 2019;105(3):581-90.

4. Trotti A, Bellm LA, Epstein JB, Frame D, Fuchs HJ, Gwede CK, et al. Mucositis incidence, severity and associated outcomes in patients with head and neck cancer receiving radiotherapy with or without chemotherapy: a systematic literature review. Radiother Oncol. 2003;66(3):253-62.

5. Wu SX, Cui TT, Zhao C, Pan JJ, Xu BY, Tian Y, et al. A prospective, randomized, multi-center trial to investigate Actovegin in prevention and treatment of acute oral mucositis caused by chemoradiotherapy for nasopharyngeal carcinoma. Radiother Oncol. 2010;97(1):113-8.

6. Luo DH, Hong MH, Guo L, Cao KJ, Deng MQ, Mo HY. Analysis of oral mucositis risk factors during radiotherapy for nasopharyngeal carcinoma patients and establishment of a discriminant model. Ai Zheng. 2005;24:850-4.

7. Nishioka T, Shirato $\mathrm{H}$, Arimoto T, Kaneko M, Kitahara T, Oomori K, et al. Reduction of radiationinduced xerostomia in nasopharyngeal carcinoma using CT simulation with laser patient marking and three-field irradiation technique. Int J Radiat Oncol Biol Phys. 1997;38(4):705-12.

8. Li G, Jiang XY, Qiu B, Shen LJ, Chen C, Xia YF. Vicious circle of acute radiation toxicities and weight loss predicts poor prognosis for nasopharyngeal carcinoma patients receiving intensity modulated radiotherapy. J Cancer. 2017;8(5):832-8.

9. Qiu C, Yang N, Tian G, Liu H. Weight loss during radiotherapy for nasopharyngeal carcinoma: a prospective study from northern China. Nutr Cancer. 2011;63(6):873-9. 
10. Zeng Q, Shen LJ, Guo X, Guo XM, Qian CN, Wu PH. Critical weight loss predicts poor prognosis in nasopharyngeal carcinoma. BMC Cancer. 2016;16:169.

11. Du XJ, Tang LL, Mao YP, Guo R, Sun Y, Lin AH, et al. Value of the prognostic nutritional index and weight loss in predicting metastasis and long-term mortality in nasopharyngeal carcinoma. J Transl Med. 2015;13:364.

12. Shen LJ, Chen C, Li BF, Gao J, Xia YF. High weight loss during radiation treatment changes the prognosis in under-/normal weight nasopharyngeal carcinoma patients for the worse: a retrospective analysis of 2433 cases. PLoS One. 2013;8(7):e68660.

13. Ho KF, Marchant T, Moore C, Webster G, Rowbottom C, Penington H, et al. Monitoring dosimetric impact of weight loss with kilovoltage $(\mathrm{kV})$ cone beam CT (CBCT) during parotid-sparing IMRT and concurrent chemotherapy. Int J Radiat Oncol Biol Phys. 2012;82:e375-82.

14. Jensen AD, Nill S, Huber PE, Bendl R, Debus J, Münter MW. A clinical concept for interfractional adaptive radiation therapy in the treatment of head and neck cancer. Int J Radiat Oncol Biol Phys. 2012;82:590-66.

15. Yao JJ, Lin L, Jin YN, Wang SY, Zhang WJ, Zhang F, et al. Prognostic value of serum Epstein-Barr virus antibodies in patients with nasopharyngeal carcinoma and undetectable pretreatment EpsteinBarr virus DNA. Cancer Sci. 2017;108(8):1640-7.

16. Edge SB, Compton CC. The American Joint Committee on Cancer: the 7th edition of the AJCC cancer staging manual and the future of TNM. Ann Surg Oncol. 2010;17:1471-4.

17. Zhou GQ, Tang LL, Mao YP, Chen L, Li WF, Sun Y, et al. Baseline serum lactate dehydrogenase levels for patients treated with intensity-modulated radiotherapy for nasopharyngeal carcinoma: a predictor of poor prognosis and subsequent liver metastasis. Int J Radiat Oncol Biol Phys. 2012;82(3):e35965.

18. Allin KH, Bojesen SE, Nordestgaard BG. Baseline C-reactive protein is associated with incident cancer and survival in patients with cancer. J Clin Oncol. 2009;27:2217-24.

19. Tang LQ, Li CF, Li J, Chen WH, Chen QY, Yuan LX, et al. Establishment and Validation of Prognostic Nomograms for Endemic Nasopharyngeal Carcinoma. J Natl Cancer Inst. 2015;108(1). doi:10.1371/journal.pone.0008660.

20. Baxi SS, Schwitzer E, Jones LW. A review of weight loss and sarcopenia in patients with head and neck cancer treated with chemoradiation. Cancers Head Neck. 2016;1:9. doi:10.1186/s41199-0160010-0.

21. Langius JA, Twisk J, Kampman M, Doornaert P, Kramer MH, Weijs PJ, et al. Prediction model to predict critical weight loss in patients with head and neck cancer during (chemo)radiotherapy. Oral Oncol. 2016;52:91-6.

22. Chen C, Fei Z, Chen L, Bai P, Lin X, Pan J. Will weight loss cause significant dosimetric changes of target volumes and organs at risk in nasopharyngeal carcinoma treated with intensity-modulated radiation therapy? Med Dosim. 2014;39(1):34-7. 
23. Kubrak C, Olson K, Jha N, Scrimger R, Parliament M, McCargar L, et al. Clinical determinants of weight loss in patients receiving radiation and chemoirradiation for head and neck cancer: a prospective longitudinal view. Head Neck. 2013;35(5):695-703.

24. Yao JJ, Zhang F, Gao TS, Zhang WJ, Lawrence WR, Zhu BT, et al. Survival impact of radiotherapy interruption in nasopharyngeal carcinoma in the intensity-modulated radiotherapy era: A big-data intelligence platform-based analysis. Radiother Oncol. 2019;132:178-87.

25. Datema FR, Ferrier MB, Baatenburg de Jong RJ. Impact of severe malnutrition on short-term mortality and overall survival in head and neck cancer. Oral Oncol. 2011;47(9):910-4.

26. O'Shea D, Cawood TJ, O'Farrelly C, Lynch L. Natural killer cells in obesity: impaired function and increased susceptibility to the effects of cigarette smoke. PLoS One. 2010;5(1):e8660.

27. Sun X, Su S, Chen C, Han F, Zhao C, Xiao W, et al. Long-term outcomes of intensity-modulated radiotherapy for 868 patients with nasopharyngeal carcinoma: an analysis of survival and treatment toxicities. Radiother Oncol. 2014;110(3):398-403.

28. Yao JJ, Qi ZY, Liu ZG, Jiang GM, Xu XW, Chen SY, et al. Clinical features and survival outcomes between ascending and descending types of nasopharyngeal carcinoma in the intensity-modulated radiotherapy era: A big-data intelligence platform-based analysis. Radiother Oncol. 2019;137:13744.

\section{Figures}


A

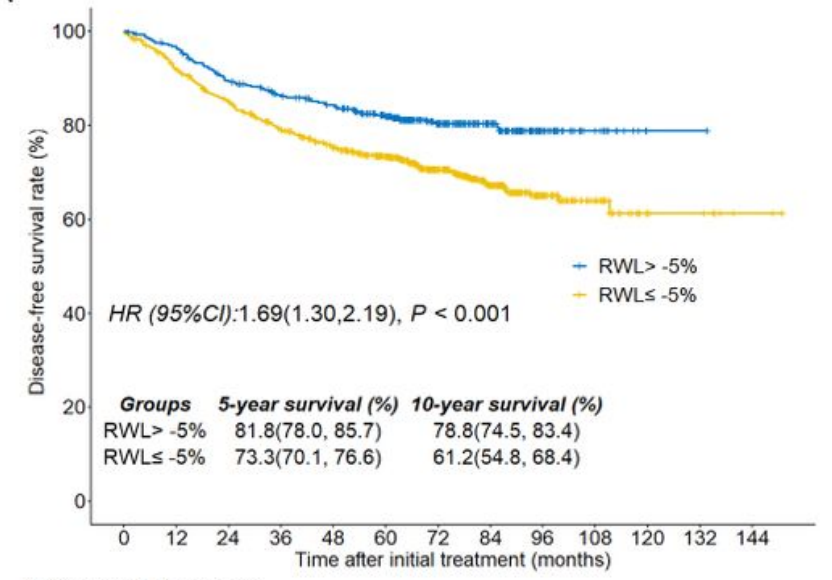
Number at risk (number censored)

- $402(0) 386(3) \quad 354(6) 332$ (16) 317 (23) $270(61) 190(138) 117(210) 39(286) 14(311) \quad 1(324) \quad 1(324) \quad 0(325$

- $747(0) 679(9) 620(17) 564(30) 516(51) 452(103) 307(232) 182(346) 76$ (448) 35 (488) $9(514) \quad 7(515) \quad 2(520)$

C

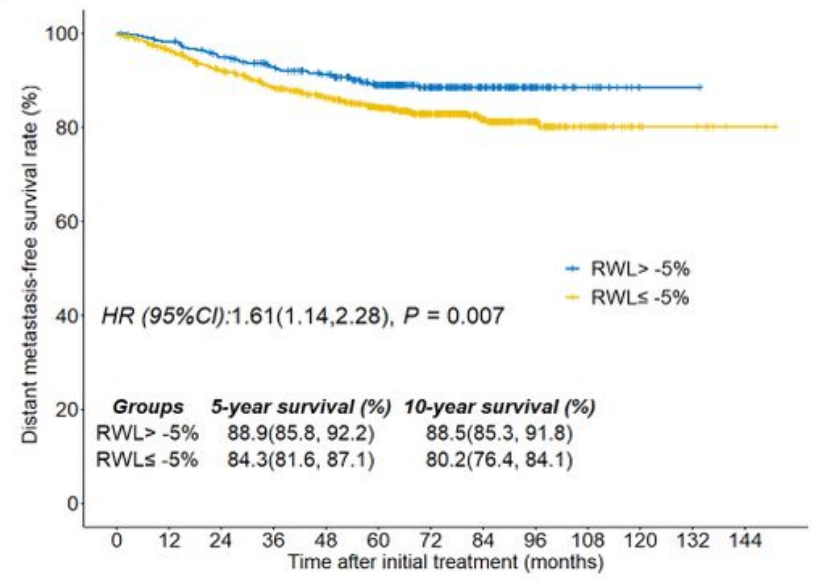
Number at risk (number censored)

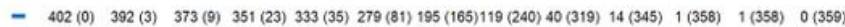

- 747 (0) $704(17) 651$ (38) $603(62) 550(100) 479(160) 322(309) 188(440) 79(548) 35(591) 9(618) \quad 7(619) \quad 2(624)$
B

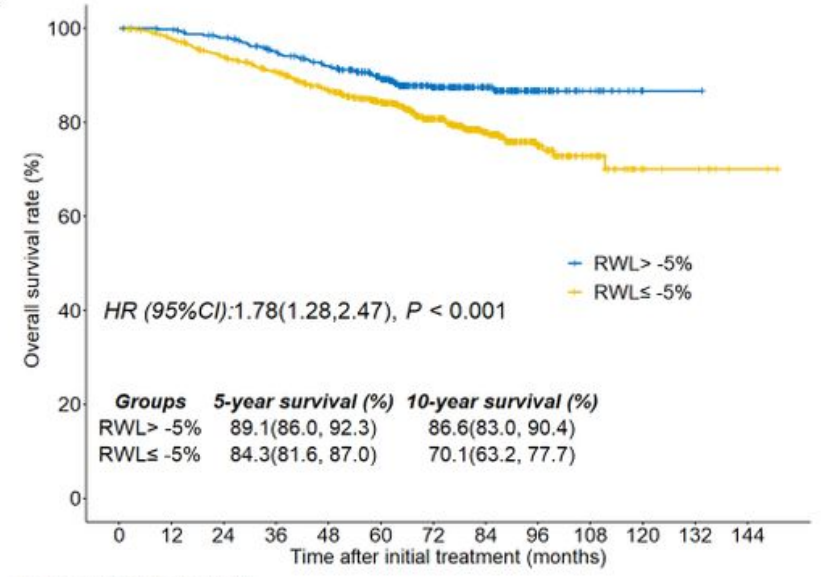
Number at risk (number censored)

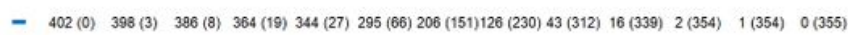

- $747(0) 722$ (9) $681(21) 642$ (38) $587(66) 505$ (132)342 (276)200 (408) 85 (518) 38 (563) 10 (591) 7 (593) 2 (598)

D

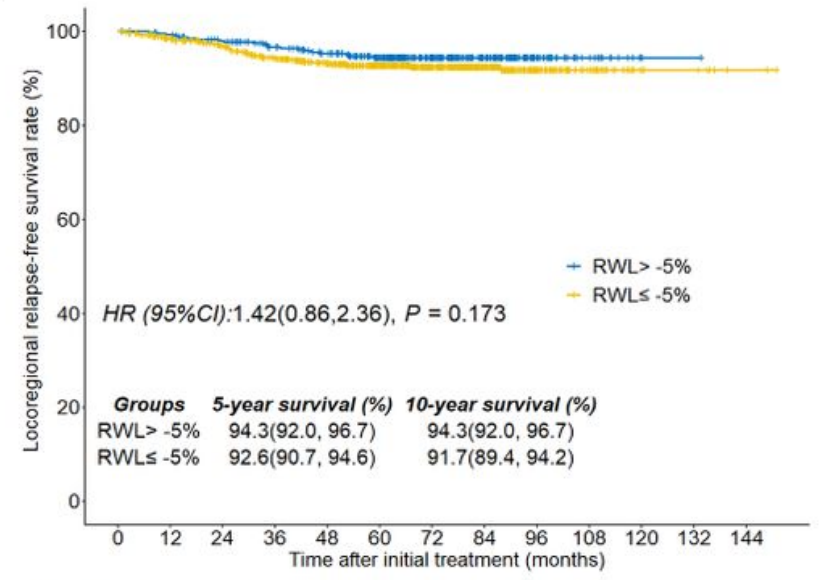
Number at risk (number censored)

- $402(0) 395(4) 379(15) 355(34) 334(50) 285(96) 200(182) 122(259) 42(339) 16(365) 2(380) \quad 1(380) \quad 0(381)$

- 747 (0) 711 (25) $665(59) 615$ (93) 561 (139)483 (215)326 (370)192 (504) 81 (614) 37 (658) 9 (687) 7 (688) 2 (693)

\section{Figure 1}

Comparison between the RWL >-5\% group and the RWL $\leq-5 \%$ group for disease-free survival (A), overall survival (B), distant metastasis-free survival (C), and locoregional relapse-free survival (D). RWL, rate of weight loss. 